\title{
Spin, gravity, and inertia
}

\author{
Yuri N. Obukhov* \\ Institute of Theoretical Physics, Technical University of Berlin, Hardenbergstr. 36, D-10623 \\ Berlin, Germany
}

\begin{abstract}
The gravitational effects in the relativistic quantum mechanics are investigated. The exact Foldy-Wouthuysen transformation is constructed for the Dirac particle coupled to the static spacetime metric. As a direct application, we analyze the non-relativistic limit of the theory. The new term describing the specific spin (gravitational moment) interaction effect is recovered in the Hamiltonian. The comparison of the true gravitational coupling with the purely inertial case demonstrates that the spin relativistic effects do not violate the equivalence principle for the Dirac fermions.
\end{abstract}

Typeset using REVTEX

*On leave from: Department of Theoretical Physics, Moscow State University, 117234 Moscow, Russia 
All high-energy physics experiments usually take place either in a curved spacetime or in a non-inertial reference frame (e.g., on Earth's surface or in the nearby space). Hence the study of the gravitational effects on quantum-mechanical systems represents an important issue. The weakness of the gravitational interaction has justified, to a certain extent, the long practice of neglecting the gravitational and/or inertial effects in particle physics.

However the technological progress, and especially the notable development of interferometric technique, has significantly changed the situation. In particular, in the famous Colella-Overhauser-Werner (COW) [1] and Bonse-Wroblewski [2] experiments, the quantummechanical phase shift due to the gravitational and inertial forces was measured, thereby verifying the validity of the equivalence principle for the non-relativistic neutron waves. The corresponding theoretical analysis was based on the Newtonian gravity and the Schrödinger equation.

It is generally believed that the further improvement of the experimental technology (using the atomic interferometers, and the polarized neutrons, e.g.) will soon provide a more precise picture of the interaction of quantum particles with the gravitational field. Under these circumstances, it seems natural to study the higher order effects in the relativistic quantum mechanics, including the specific manifestations of the spin-gravity coupling.

In this connection, it is worthwhile to recall that certain theoretical models predicted the violation of the equivalence principle for spinning particles, see e.g. [3 3 [ [5 . A good example is provided by the model of Peres [A], in which the non-relativistic Hamiltonian of a massive Dirac particle included the additional term $k \hbar c^{-1} \vec{\sigma} \cdot \vec{g}$. This describes the gravitational dipole type interaction of spin $\vec{\sigma}$ and the gravitational acceleration vector $\vec{g}$ with the dimensionless coupling constant $k$. Similar interactions were considered very early by Kobzarev and Okun, and by Leitner and Okubo [6]. The comparison with the precision experimental data places though very weak restrictions on the value of the coupling constant $k$, see the review in [5].

In contrast to the ad hoc Peres' type approach, here we will consider the standard theory of Dirac fermions in curved spacetime [7:8]. Correspondingly, the gravitationally coupled 4-spinor field $\psi$ satisfies the covariant Dirac equation:

$$
\left(i \hbar \gamma^{\alpha} D_{\alpha}-m c\right) \psi=0 .
$$


Here, $D_{\alpha}$ is the spinor covariant derivative with [9]

$$
D_{\alpha}=h_{\alpha}^{i} D_{i}, \quad D_{i}:=\partial_{i}+\frac{i}{4} \widehat{\sigma}_{\alpha \beta} \Gamma_{i}^{\alpha \beta}
$$

We use the conventions of Bjorken and Drell [10] for the Dirac matrices $\gamma^{\alpha}, \beta, \vec{\alpha}$; as usual, $\widehat{\sigma}^{\alpha \beta}:=i \gamma^{[\alpha} \gamma^{\beta]}$. Gravitational and inertial effects are encoded in the (co)frame and the Lorentz connection coefficients

$$
h_{i}^{\alpha}, \quad \Gamma_{i}^{\alpha \beta}=-\Gamma_{i}^{\beta \alpha} .
$$

As it is well known, at any point $P$, it is always possible to choose the local spacetime coordinates and the (non-holonomic, in general) frame so that $h_{i}^{\alpha}(P)=\delta_{i}^{\alpha}, \Gamma_{i}^{\alpha \beta}(P)=0$. This mathematical fact underlies the equivalence principle in accordance with which the gravitationally coupled Dirac equation (1) locally (at every point) assumes its flat space form in a suitably chosen reference frame 8$]$.

The dynamics of the Dirac fermions in the different gravitational fields and non-inertial reference frames was studied previously [11] using approximation schemes. Here we for the first time will present some exact results. More specifically, let us confine our attention to the wide class of spacetimes described by the static metric

$$
d s^{2}=V^{2}\left(d x^{0}\right)^{2}-W^{2}(d \vec{x} \cdot d \vec{x})
$$

where $x^{0}=c t$, and $V=V(\vec{x}), W=W(\vec{x})$ are the arbitrary functions of the spatial coordinates $\vec{x}$. Two important particular cases belong to this family: (i) the flat Minkowski spacetime in accelerated frame:

$$
V=1+\frac{(\vec{a} \cdot \vec{x})}{c^{2}}, \quad W=1
$$

and (ii) Schwarzschild spacetime in isotropic coordinates:

$$
V=\left(1-\frac{G M}{2 c^{2} r}\right)\left(1+\frac{G M}{2 c^{2} r}\right)^{-1}, \quad W=\left(1+\frac{G M}{2 c^{2} r}\right)^{2}
$$

with $r:=\sqrt{\vec{x} \cdot \vec{x}}$. Choosing the orthonormal frame,

$$
h_{i}^{\widehat{0}}=V \delta_{i}^{0}, \quad h_{i}^{\widehat{a}}=W \delta_{i}^{a}, \quad a, b=1,2,3,
$$

we find the local Lorentz connection: 


$$
\Gamma_{i}^{\widehat{a} \widehat{0}}=\frac{\partial^{a} V}{W V} h_{i}^{\widehat{0}}, \quad \Gamma_{i}^{\widehat{a} \widehat{b}}=\frac{\partial^{a} W}{W^{2}} h_{i}^{\widehat{b}}-\frac{\partial^{b} W}{W^{2}} h_{i}^{\widehat{a}} .
$$

Hereafter, the hats distinguish the local frame indices from the spacetime coordinate ones. As a result, we have the explicit spinor derivative components [12]:

$$
\begin{aligned}
& D_{\widehat{0}}=\frac{1}{V}\left(\frac{\partial}{\partial x^{0}}+\frac{1}{2 W}(\vec{\alpha} \cdot \vec{\nabla} V)\right), \\
& D_{\widehat{a}}=\frac{1}{W}\left(\frac{\partial}{\partial x^{a}}+\frac{i}{2 W} \epsilon_{a b c} \partial^{b} W \Sigma^{c}\right) .
\end{aligned}
$$

Consequently, the Dirac equation (1) is recasted into the familiar Schrödinger form

$$
i \hbar \frac{\partial \psi}{\partial t}=\widehat{\mathcal{H}} \psi
$$

with the Hamilton operator

$$
\begin{aligned}
\widehat{\mathcal{H}}= & \beta m c^{2} V+\frac{V}{W} c(\vec{\alpha} \cdot \vec{p}) \\
& -\frac{i \hbar c}{2 W}(\vec{\alpha} \cdot \vec{\nabla} V)-\frac{i \hbar c V}{W^{2}}(\vec{\alpha} \cdot \vec{\nabla} W) .
\end{aligned}
$$

Redefining the spinor field and the Hamiltonian,

$$
\psi^{\prime}=W^{3 / 2} \psi, \quad \widehat{\mathcal{H}^{\prime}}=W^{3 / 2} \widehat{\mathcal{H}} W^{-3 / 2},
$$

we obtain the new Hamiltonian (which is explicitly Hermitian with respect to the usual flat space scalar product):

$$
\widehat{\mathcal{H}^{\prime}}=\beta m c^{2} V+\frac{c}{2}[(\vec{\alpha} \cdot \vec{p}) \mathcal{F}+\mathcal{F}(\vec{\alpha} \cdot \vec{p})],
$$

where $\mathcal{F}:=V / W$. From now on we will drop the prime.

In order to reveal the true physical content of the theory and to obtain its correct interpretation, it is necessary to perform the Foldy-Wouthuysen (FW) transformation [13, uncoupling the positive and the negative energy states. The corresponding unitary operator can be easily obtained for the free Dirac particle. But in most cases for a fermion interacting with an electromagnetic field, there is no exact transformation. Instead, the approximate scheme is used in which the odd parts of Hamiltonian are removed order by order in powers of $\left(m c^{2}\right)^{-1}$ [10]. The same approximations method was also applied in all the previous studies of the gravitational effects [11]. 
However quite remarkably, one can construct the exact $F W$ transformation for the Dirac particle moving in the metric (田) under consideration. The main guidelines are provided by Eriksen's approach to the FW transformation [14]. The key idea is to construct the unitary operator $U$, relating the FW-representation to the Dirac-representation $\psi^{F}=U \psi$, which satisfies the condition

$$
U \widehat{\Lambda} U^{\dagger}=\beta .
$$

Here

$$
\widehat{\Lambda}=\widehat{\mathcal{H}} / \sqrt{\widehat{\mathcal{H}^{2}}}
$$

is Pauli's [15] sign energy operator. By definition, it is Hermitian, unitary, and idempotent: $\widehat{\Lambda}^{2}=\widehat{\Lambda}^{\dagger} \widehat{\Lambda}=1$ (as usually, one should assume that Hamiltonian is well defined in that it does not possess zero eigenvalues, see [14]).

It is now crucial to observe that the Hamiltonian (14) admits the anticommuting involution operator

$$
J:=i \gamma_{5} \beta
$$

Clearly, this operator is Hermitian, $J^{\dagger}=J$, and unitary, $J J^{\dagger}=J^{2}=1$. It anticommutes both with the Hamiltonian (14) and with the $\beta$ matrix:

$$
J \widehat{\mathcal{H}}+\widehat{\mathcal{H}} J=0, \quad J \beta+\beta J=0 .
$$

Now, it is straightforward to see that the FW transformation is realized by means of the operator $U=U_{2} U_{1}$, where

$$
U_{1}=\frac{1}{\sqrt{2}}(1+J \widehat{\Lambda}), \quad U_{2}=\frac{1}{\sqrt{2}}(1+\beta J) .
$$

Indeed, we immediately find

$$
U_{1} \widehat{\Lambda} U_{1}^{\dagger}=J, \quad U_{2} J U_{2}^{\dagger}=\beta
$$

and consequently (19) satisfies the FW condition (15).

The final step is to find the Hamiltonian $\widehat{\mathcal{H}}^{F}=U \widehat{\mathcal{H}} U^{\dagger}$ in the FW-representation. From (18) we have $J \widehat{\mathcal{H}}^{2}=\widehat{\mathcal{H}}^{2} J$, hence $J \sqrt{\widehat{\mathcal{H}}^{2}}=\sqrt{\widehat{\mathcal{H}}^{2}} J$, and $J \widehat{\Lambda}+\widehat{\Lambda} J=0$. Consequently, one finds that $U_{1} \widehat{\mathcal{H}} U_{1}^{\dagger}=J \sqrt{\widehat{\mathcal{H}^{2}}}$, and finally, 


$$
U \widehat{\mathcal{H}} U^{\dagger}=U_{2} U_{1} \widehat{\mathcal{H}} U_{1}^{\dagger} U_{2}^{\dagger}=\left[\sqrt{\widehat{\mathcal{H}}^{2}}\right] \beta+\left\{\sqrt{\widehat{\mathcal{H}^{2}}}\right\} J
$$

Here, as usually, the even and odd parts of any operator $Q$ are defined as

$$
[Q]:=\frac{1}{2}(Q+\beta Q \beta), \quad\{Q\}:=\frac{1}{2}(Q-\beta Q \beta) .
$$

Note that both terms in (21) are clearly even, thus indeed the FW Hamiltonian does not mix the upper and lower spinor components (i.e., positive and negative energy states).

Usually (at least in the known cases when the exact FW transformation exists for the electromagnetic coupling), the square of Hamiltonian turns out to be an even operator, and then the second term in (21) is absent. However, this is not the case for our problem, because the square of Hamilton operator

$$
\begin{aligned}
\widehat{\mathcal{H}}^{2}= & m^{2} c^{4} V^{2}+\mathcal{F} c^{2} p^{2} \mathcal{F}+\frac{\hbar^{2} c^{2}}{2} \mathcal{F}(\vec{\nabla} \cdot \vec{f})-\frac{\hbar^{2} c^{2}}{4} \vec{f}^{2} \\
& +\hbar c^{2} \mathcal{F} \vec{\Sigma} \cdot([\vec{f} \times \vec{p}]+J m c \vec{\phi})
\end{aligned}
$$

contains the odd piece (the very last term in the above expression). Here we denoted the gradients

$$
\vec{\phi}:=\vec{\nabla} V, \quad \vec{f}:=\vec{\nabla} \mathcal{F}
$$

The FW Hamiltonian (21) is exact. Now we may turn to its analysis, looking for different important limiting cases. For the most practical purposes, it is sufficient to use the non-relativistic wave functions, treating all the interaction terms as perturbations. The quasi-relativistic approximation is straightforwardly obtained by assuming that $m c^{2}$ term is dominating, and thus correspondingly expanding the square root of (23) as

$$
\begin{aligned}
\sqrt{\widehat{\mathcal{H}}^{2}} \approx & m c^{2} V+\frac{1}{4 m}\left(W^{-1} p^{2} \mathcal{F}+\mathcal{F} p^{2} W^{-1}\right)+\frac{\hbar^{2}}{4 m W}(\vec{\nabla} \cdot \vec{f})-\frac{\hbar^{2}}{8 m V} \vec{f}^{2} \\
& +\frac{\hbar}{4 m} \vec{\Sigma} \cdot\left(W^{-1}[\vec{f} \times \vec{p}]+[\vec{f} \times \vec{p}] W^{-1}+J 2 W^{-1} m c \vec{\phi}\right)
\end{aligned}
$$

with the subsequent extraction of the even and odd pieces according to (22). It seems worthwhile to note the appearance of the "gravitational Darwin" term

$$
\frac{\hbar^{2}}{4 m W}(\vec{\nabla} \cdot \vec{f})=\frac{\hbar^{2}}{4 m W} \Delta \mathcal{F}
$$


which was not reported before [11. It clearly admits a physical interpretation similar to that of the usual electromagnetic Darwin term, reflecting the zitterbewegung fluctuation of the fermion's position with the mean square $<(\delta r)^{2}>\sim \hbar^{2} /(m c)^{2}$. Analyzing the effective gravitostatic energy instead of the electrostatic one (with the charge $e$ replaced by the mass $m)$, one then can derive a contribution of the form (26).

In order to compare the relativistic spin effects of the gravitational and inertial forces, and thereby to obtain an insight into the validity of the equivalence principle for fermions, it is instructive to consider separately the two above mentioned particular cases (5) and (6) of the metric (四).

(i) Accelerated frame. From (5) we find $\mathcal{F}=V$, and consequently:

$$
\vec{\phi}=\vec{f}=\frac{\vec{a}}{c^{2}}
$$

Preserving the leading contributions, we then find for the non-relativistic FW Hamiltonian:

$$
\begin{aligned}
\widehat{\mathcal{H}}^{F}= & \beta m c^{2}+\beta m \vec{a} \cdot \vec{x}+\beta \frac{p^{2}}{2 m}+\frac{\hbar}{2 c} \vec{\Sigma} \cdot \vec{a} \\
& +\frac{\hbar}{2 m c^{2}} \beta \vec{\Sigma} \cdot[\vec{a} \times \vec{p}] .
\end{aligned}
$$

The Darwin term identically vanishes for obvious reasons.

(ii) Spherically symmetric gravitational field. Far away from the central gravitating body of a mass $M$, it is sufficient to consider a weak limit of the Schwarzschild field (6) which yields

$$
V \approx 1-\frac{G M}{c^{2} r}, \quad W \approx 1+\frac{G M}{c^{2} r}
$$

Correspondingly,

$$
\vec{\phi}=-\frac{\vec{g}}{c^{2}}, \quad \vec{f}=-\frac{2 \vec{g}}{c^{2}}, \quad \text { with } \quad \vec{g}=-G M \frac{\vec{r}}{r^{3}}
$$

and the non-relativistic FW Hamiltonian reads:

$$
\begin{aligned}
\widehat{\mathcal{H}}^{F}= & \beta m c^{2}+\beta m \vec{g} \cdot \vec{x}+\beta \frac{p^{2}}{2 m}-\frac{\hbar}{2 c} \vec{\Sigma} \cdot \vec{g} \\
& -\frac{\hbar}{m c^{2}} \beta \vec{\Sigma} \cdot[\vec{g} \times \vec{p}]-\frac{\hbar^{2} \beta}{2 m}(\vec{\nabla} \cdot \vec{g}) .
\end{aligned}
$$

In both cases we neglect in (28) and (31) the higher order relativistic and gravitational/inertial ("red shift" etc) terms. 
The most nontrivial common feature is the recovery of the Peres' type spin contribution [last terms on the first lines in (28) and (31)]. We have however two important differences from [4]. Firstly, unlike the ad hoc model of Peres, the corresponding coupling constant is now fixed: $k=1 / 2$. Secondly, the same spin term is present in both cases, one just needs to replace the acceleration $\vec{a}$ of a reference system by the true gravitational acceleration $\vec{g}$. Hence, in contrast to Peres' model, the covariant Dirac theory proves the validity of the equivalence principle, also with the higher order relativistic spin effects taken into account.

One can estimate (following [4) the influence of the additional term. For the gravitational field of the Earth one has $g / c=3.271 \times 10^{-8} \mathrm{~Hz}$, or $\hbar g / c=2.153 \times 10^{-23} \mathrm{eV}$, which is essentially smaller than what the present experimental technique can detect [the next terms in (28) and (31) are several orders weaker]. At the same time, this is also in a good agreement with the strong limits set by the high precision measurements, e.g., of the hyperfine splitting, [6]. A possible direct test might be carried out with a spin-polarized macroscopic body [16]. A comment is needed for explaining the difference of our derivations with the earlier results [11. On the one hand, it is important to realize that the approximate scheme [10] developed for the case of electromagnetic coupling is not, strictly speaking, applicable in the gravitational interaction case. The idea of such an approximate scheme is to remove, order by order in $1 / m$, the odd terms from the Hamiltonian $\widehat{\mathcal{H}}=\beta m c^{2}+\mathcal{E}+\mathcal{O}$. Normally, the odd $\mathcal{O}$ and even $\mathcal{E}$ parts did not depend on the mass $m$ : All the terms were proportional to the electromagnetic charge $e$, and that made the standard scheme [10 working. However, for the gravitational/inertial case, the even part $\mathcal{E}$ necessarily contains the terms proportional to the gravitational/inertial charge $m$. As a result, although in the first approximation the original odd term $\mathcal{O}$ is removed, the new term is produced $\beta[\mathcal{O}, \mathcal{E}] /(2 m)$ which gives a contribution of order $m^{0}$. The same is repeated at every step of the approximate scheme, so that the remaining even terms are always of the same order in $1 / m$ as the "removed" odd terms. This makes the crucial issue of convergence of the approximation scheme problematic [18]. In our approach, we avoid this deficiency by using the exact $\mathrm{FW}$ transformation. It seems worthwhile to notice that the method works also for the case with the magnetic field coupling included, when a generalization of the result of Case [17 is obtained. On the other hand, the FW transformation is defined with a certain ambiguity. The unitary transformation 
$U=e^{i S}$ with $S=-\beta /(4 m c) \vec{\Sigma} \cdot\left(W^{-1} \vec{p}+\vec{p} W^{-1}\right)$ brings the Hamiltonian $\widehat{\mathcal{H}^{F}} \rightarrow U \widehat{\mathcal{H}^{F}} U^{\dagger}$ to the approximate form reported by Fischbach et al and by Hehl and Ni [11].

A spin carried by a fermion can be visualized (with the obvious reservations and a reasonable portion of caution [19]) as some sort of intrinsic circular motion. In this primitive picture, a particle's electric charge induces an Ampère type ring current which, in turn, according to the Oersted-Ampère law, acts as a magnetic moment. The massive fermion, besides the electric charge, carries also the gravitational charge. Accordingly, in the framework of general relativity, one can naturally expect a mass-energy ring current inducing a gravitational moment [20]. In this paper we have demonstrated how the gravitational moment can show up explicitly in the non-relativistic limit of the covariant Dirac theory.

Acknowledgments. This work was supported by the Deutsche Forschungsgemeinschaft (Bonn). 


\section{REFERENCES}

[1] R. Colella, A.W. Overhauser, and S.A. Werner, Phys. Rev. Lett. 34 (1975) 1472.

[2] U. Bonse and T. Wroblewski, Phys. Rev. Lett. 51 (1983) 1401.

[3] T.A. Morgan and A. Peres, Phys. Rev. Lett. 9 (1962) 79; W.-T. Ni, Phys. Rev. Lett. 38 (1977) 301; N.D. Hari Dass, Phys. Rev. Lett. 36 (1976) 393; N.D. Hari Dass, Ann. Phys. (NY) 107 (1977) 337.

[4] A. Peres, Phys. Rev. D18 (1978) 2739.

[5] B. Mashhoon, Class. Quantum Grav. 17 (2000) 2399.

[6] I.Yu. Kobzarev and L.B. Okun, Sov. Phys. JETP 16 (1963) 1343 [ZhETF 43 (1962) 1904 (in Russian)]; J. Leitner and S. Okubo, Phys. Rev. 136 (1964) 1542.

[7] V.A. Fock and D.D. Ivanenko, C.R. Acad. Sci. Paris 188 (1929) 1470; V.A. Fock and D.D. Ivanenko, Z. Phys. 54 (1929) 798; H. Tetrode, Z. Phys. 50 (1928) 336; V. Bargmann, Sitzungsber. preuss. Akad. Wiss. Phys.-math. Kl. (1932) 346; E. Schrödinger E., Sitzungsber. preuss. Akad. Wiss. Phys.-math. Kl. (1932) 105; L. Infeld L. and B.L. van der Waerden, Sitzungsber. preuss. Akad. Wiss. Phys.-math. Kl. (1933) 380; 474; D.R. Brill and J.A. Wheeler, Rev. Mod. Phys. 29 (1957) 465, Errata: Rev. Mod. Phys. 33 (1961) 623.

[8] See also a general discussion in: F.W. Hehl, J. Lemke, and E.W. Mielke, Two lectures on fermions and gravity, in: Geometry and Theoretical Physics, Proc. of the Bad Honnef School 12-16 Feb. 1990, J. Debrus and A.C. Hirshfeld, eds. (Springer: Heidelberg, 1991) pp. $56-140$.

[9] We use the Greek alphabet for the indices which label the components with respect to a local Lorentz frame $e_{\alpha}=h_{\alpha}^{i} \partial_{i}$, whereas the Latin indices refer to the local spacetime coordinates $x^{i}$.

[10] J.D. Bjorken and S.D. Drell, Relativistic Quantum Mechanics (McGraw-Hill: San Francisco, 1964). 
[11] C.G. de Oliveira and J. Tiomno, Nuovo Cim. 24 (1962) 672; J. Audretsch and G. Schäfer, Gen. Rel. Grav. 9 (1978) 243; E. Fischbach, B.S. Freeman, and W.-K. Cheng, Phys. Rev. D23 (1981) 2157; F.W. Hehl and W.-T. Ni, Phys. Rev. D42 (1990) 2045; Y.Q. Cai and G. Papini, Phys. Rev. Lett. 66 (1991) 1259; 68 (1992) 3811; J.C. Huang, Ann. d. Phys. 3 (1994) 53; K. Konno and M. Kasai, Prog. Theor. Phys. 100 (1998) 1145; K. Varjú and L.H. Ryder, Phys. Lett. A250 (1998) 263; L. Ryder, J. Phys. A: Math. Gen. A31 (1998) 2465. K. Varjú and L.H. Ryder, Phys. Rev. D62 (2000) 024016.

[12] As usually, we have $\beta=\gamma^{\widehat{0}}, \vec{\alpha}=\beta \vec{\gamma}, \gamma_{5}=-i \gamma^{\widehat{0}} \gamma^{\widehat{1}} \gamma^{2} \gamma^{3}$. The spin matrix is $\vec{\Sigma}=i \vec{\gamma} \times \vec{\gamma} / 2=$ $-\gamma_{5} \vec{\alpha}=\left(\begin{array}{cc}\vec{\sigma} & 0 \\ 0 & \vec{\sigma}\end{array}\right)$.

[13] L.L. Foldy and S.A. Wouthuysen, Phys. Rev. 78 (1950) 29.

[14] E. Eriksen and M. Kolsrud, Nuovo Cim. 18 (1960) 1; A.G. Nikitin, J. Phys. A: Math. Gen. A31 (1998) 3297.

[15] W. Pauli, Die allgemeinen Prinzipien der Wellenmechanik, in: Handbuch der Physik, Ed. S. Flügge (3. Aufl., Berlin, 1958) Bd. 5, Teil 1, S. 1-168.

[16] An idea of one such experiment, as proposed in [4], is to observe a breaking of the equilibrium of a polarized body, hanging in the gravitational field, when its polarized state is destroyed. The main difficulty here is to provide a very high degree of shielding of external magnetic fields.

[17] K.M. Case, Phys. Rev. 95 (1954) 1323.

[18] The danger of a failure of convergence in presence of terms of positive orders in $m$ is discussed, e.g., by J.P. Costella and B.H.J. McKellar, Am. J. Phys. 63 (1995) 1119.

[19] See, though, the careful analysis by T.T. Chou and C.N. Yang, Nucl. Phys. B107 (1976) 1, who conclude that "a polarized Dirac electron is a rotating particle" which carries the electric current.

[20] The notion of the gravitational moment was proposed in [6]. For the recent studies, see F.W. Hehl, A. Macias, E.W. Mielke, and Yu.N. Obukhov, in: "On Einstein's path" 
Festschrift for E. Schucking. A. Harvey, ed. (Springer: New York, 1999) 255-274; Yu.N. Obukhov, Acta Phys. Polon. B29 (1998) 1131. 